\title{
A Study on Energy Model Prototyping in Various Simulators of WSN
}

\author{
M. Thangaraj ${ }^{1}$ and S.Anuradha ${ }^{2}$ \\ I'Associate Professor, Department of Computer Science, Madurai Kamaraj University, India) \\ ${ }^{2}$ (Research Scholar, Department of Computer Science, Madurai Kamaraj University, India)
}

\begin{abstract}
Simulators are used to abstract the real hardware behaviors and proceed the research more economically. Various simulators of Wireless Sensor Network (WSN) are used to access the performance and transmission processes involved in WSN. Each of the simulators has its own model to support the protocol stacks of physical layer and data layer, routing algorithms, topology control. This decade issue of Energy Engineering in WSN is getting the growing attention now days. This paper is to study and compare the various Energy Model support in the simulators of WSN with the following details of features, configuration setup, data structures, result traces, monitor outputs, debugging facilities, libraries and utilities and GUI support to explain the usage. Wide range of simulators is considered for the study; the classification of simulators with the key features is briefed with their model architectures. This paper will be the unique guide for any researcher who works on the energy relevant studies in WSN field to choose the proper tool and learn its usage and architecture with minimal learning curve.
\end{abstract}

Keywords: Wireless Sensor Networks, Energy Model, Power Spectrum, Simulator, Test bed, Emulator

\section{Introduction}

It is highly expensive to study the wireless sensor network behavior in the real infrastructure setup. Like any other discipline WSN behaviors are also depicted in various simulation mediums. WSN has its applications in different domains like health care, environment study, military, intelligent homes, infrastructure management, and Traffic intelligence system. The initial level application development and study of performance in diverse WSN system with their distinguishing features like topology, propagation delays, transmission methods, data aggregations, data packet details, algorithms used to determine routing, different communication layers need the simulation system and test beds for validating and testing[1]. Ample studies are getting conducted by the academicians, research groups and the industry experts in this discipline. The critical part is to choose the right simulation platform for the research. Usually, the decisions are taken by considering the models supported (nodes, communication layers, events, power etc.) and the features provided (tracing, GUI support, Configuration possibilities, shortcoming, debugging, graph generation facilities) with any simulators. The second part is to minimize the learning curve of the simulators for the researchers[2].

This paper is going to be the guide post for the WSN researchers to choose the simulation medium for their research in the energy engineering discipline. This paper attempts to classify clearly the simulators along with the list of features and architecture of simulators' models for better understanding. The limitations are discussed in detail to help the choice for the researcher's study. At the initial stage of WSN, the simulators are extended from the conventional network simulators but with the support of the WSN specific protocols and mechanisms of sensors like NS2. Now a days WSN specific simulators are getting released where the clearly defined WSN architecture is depicted in the simulation medium as well like SensorSim, SENSE. Herewith we first define the various simulation mediums and classify them [3].

\subsection{Simulators}

\section{Simulation Medium}

Simulators are the software that models the different WSN components of the real world. It imitates the real world scenario by software components. As most of the application interfaces are exposed as API (Application Program Interfaces), almost all the simulators are extendable for the addition of features. This helps for the research and initial level of application development or investigation of performance or transmission propagation delays with minimum effort and cost.

\subsection{Emulators}

The emulators are the simulation mediums where the application code is written for the specific hardware units of WSN. The hardware nodes, gate ways and channel are specific to the vendor. The tricky part is to choose the right interface for the concerned hardware. Vendors like Zig bee, National instruments provide 
their driver interfaces and client APIs for their own hardware which can be added as a plug in to the simulation software.

\subsection{Test Beds}

It is a platform equipped with WSN infrastructure under working condition. Usually, academic institutions or Product Company ties up with government agencies and establishes the infrastructure and make it available with the software interface for the academic research purpose. The students can book the infrastructure for a time period and avail the services for the booked times lot remotely.

\subsection{Classification of Simulators}

Based on the considered environment of the WSN profile, data involved, the system behavior, preferences and purpose of the research, features provided the WSN simulators are classified as follows[4].

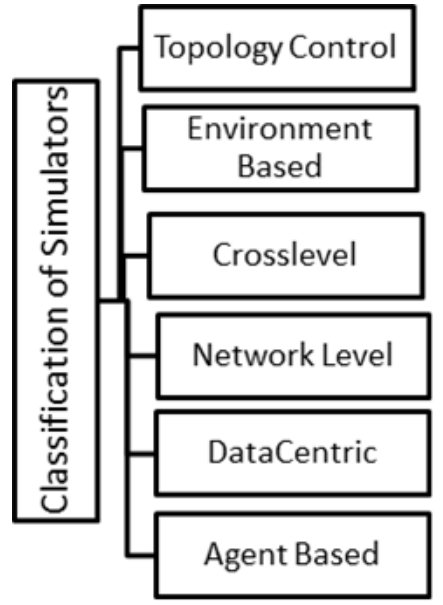

\subsection{Features Summary}

Figure 1: Classification of Simulators

The following simulators are considered for the evaluation and support for the energy study on WSN. JIST, SSFNET, NCTUn2.0, JSIM, Ptolemyii, Cooja, NesCT, PAW is, TOSSIM, EMTOS, ATEMU, DaSSF, SQualnet, OPNET, Shawn, SIDNET, SWAN, Sinalgo, WSM, Shox, TRMSim WSN, WSNSimpy, C-Sense, SENSE, S3,Avrora, Freemote, Algosensim, Atarraya, Net Topo, VMNET, MATLAB, NS3, WSNET, OMNET++. Most of the research starts with the goal in focus but leaving the detail evaluation of the research path. After a major progress in the implementation on the desired concepts, we realize the tool is not capable enough to complete the work ahead or show case the result[5].

The battery model or power framework or the power spectrum as the different simulators name the energy model of the WSN, should be adept to study the energy usage at the different layers of WSN. To choose the right simulator, define the area, scope of the research or development. Decide about the output reports and graphs. Based on that explore and evaluate the simulators to choose the right simulation medium[1].

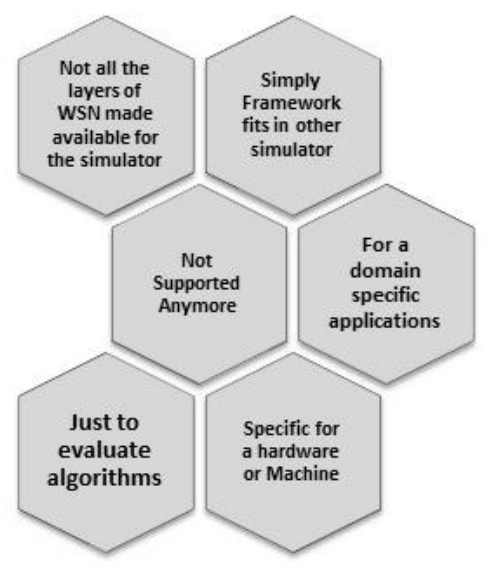

Figure 2:Factors considered

The operating system in which the simulator run, whether it has multiple platform support matters when we consider the choice of the simulator. The simulator's performance matrix covers the transmission time, 
throughput, delay, sensor coverage distance, life time etc. Here the decision of the simulator for the research depends on whether we can generate the matrix of our choice directly or from the log data.

Some of the simulators provide the probabilistic representation of the transmission errors and the delays which may not be similar to the real world environment[4].

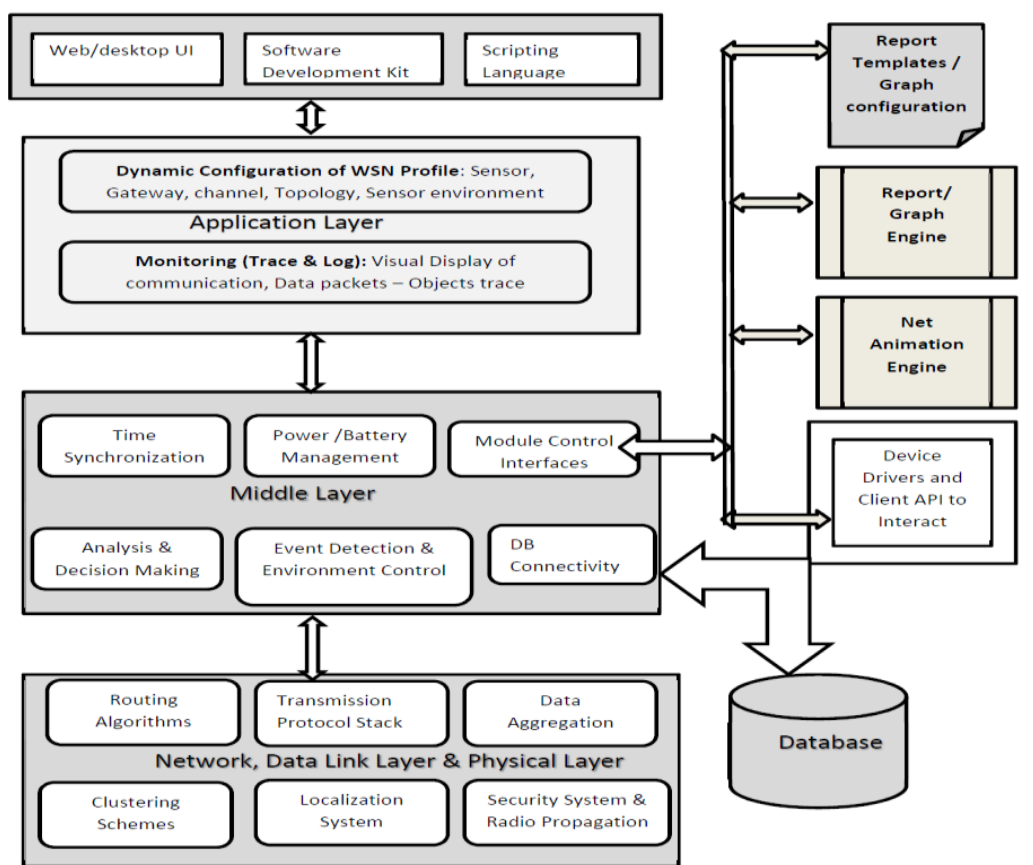

Figure 3: Simulator Architecture

It is the generic architecture of simulators. It varies for each simulator based on the purpose and the platform it is designed for [6].

III. Features And Architecture

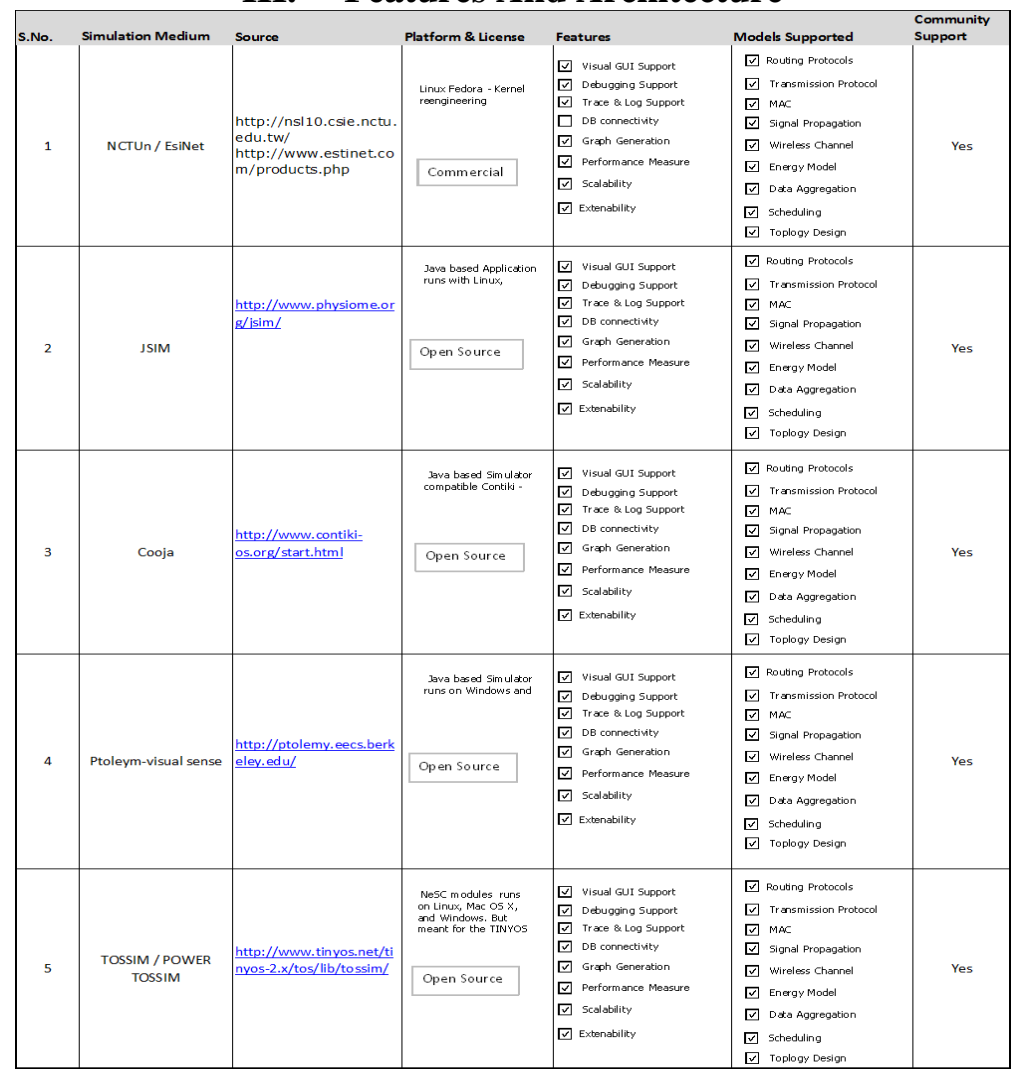




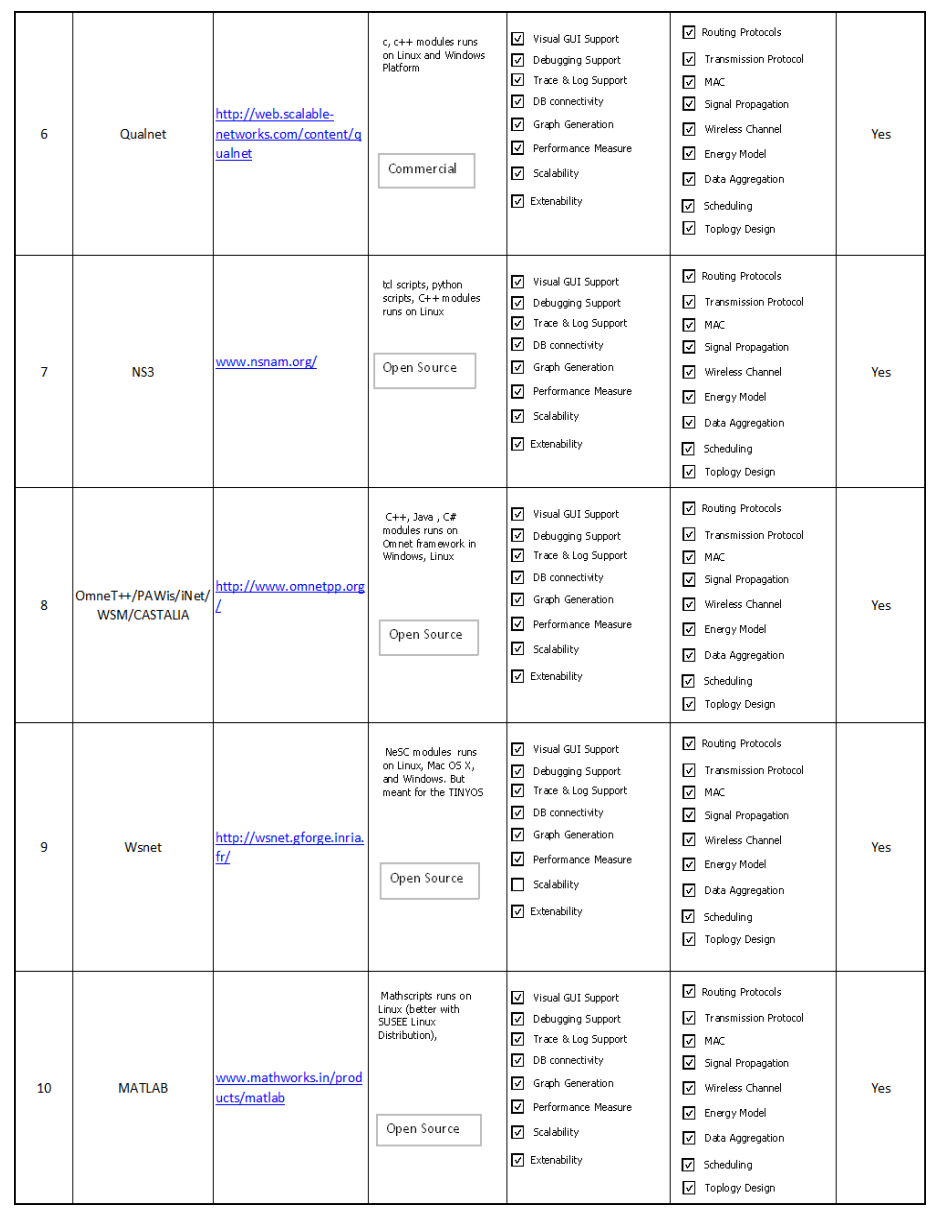

\section{Basic Concepts}

Simulators are becoming popular based on the easiness of use and the strong programming model support. It really helps the researcher or application developer to focus only on the research issue not on the simulator features[5]. For eg. if the simulator does not support the Database connectivity feature and uses only files to rage, the researcher has to develop a DB connectivity drive, which is not his research scope. If the simulator doesn't support the graph or visual animation of WSN communications, it is going to add the over head of plugging in a third party compatible graph tool or visualizer to the simulator. This session details out the approach of the simulators on basic concepts [3].

\subsection{Process Flow}

The simulator support must extend help to the developer to write the simulation code as process sequences in the Work Flow Frameworks. Means, sometimes for the business users, the simulator must be able to abstract the process flow details and help them to flow chart the processes what they want to simulate.

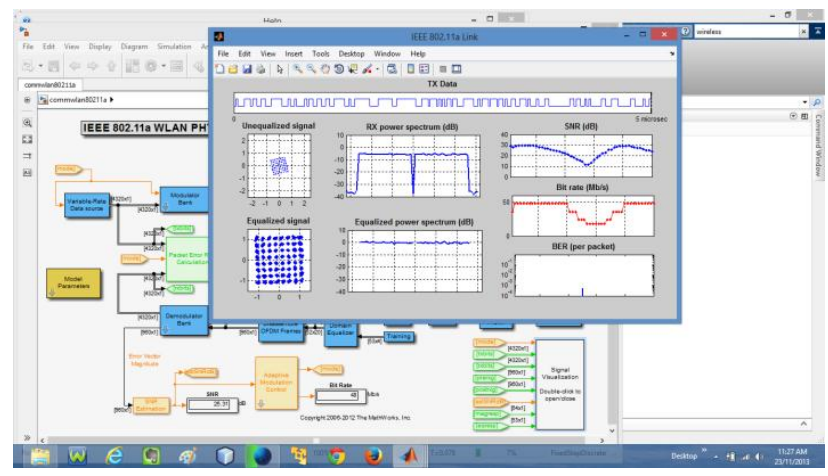

Figure 4: Power Spectrum Graph Generated in MATLAB 


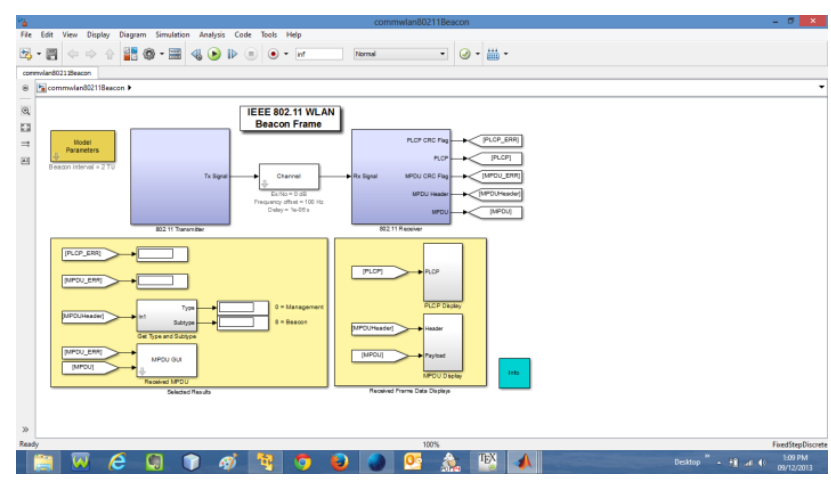

Figure 5: Designing the Process Flow in MATLAB

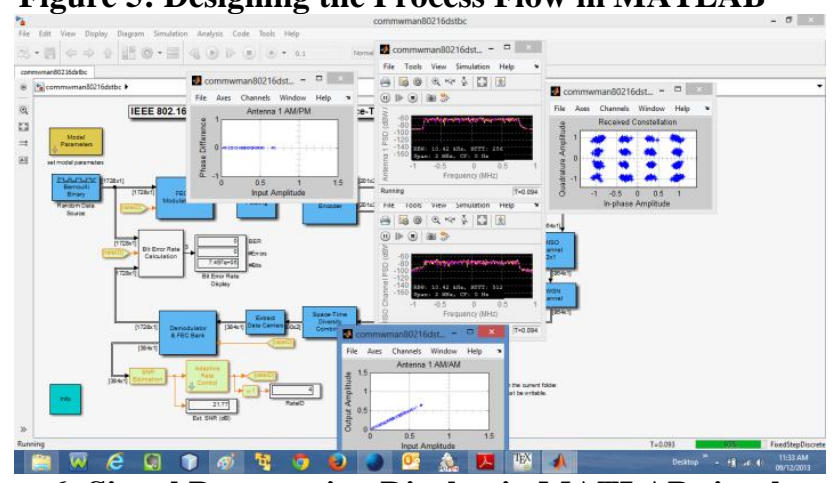

Figure 6: Signal Propagation Display in MATLAB simulator

\subsection{Signal Propagation}

The received power signals varies because of the radio channel uncertainty and the environment of the research whether it is indoor or outdoor. There are other factors like localization algorithms used can also affect the signal strength. The simulator must allow the developer to view and control the signal propagation[5].

\subsection{Data Aggregation}

The sensor nodes are of limited sensing capabilities and when it is getting forwarded, the data will be insufficient for the decision. So, data from different nodes are aggregated and forwarded to make the decisions. It reduces the redundancy and the frequent transmission. To carry out the aggregation logically the sensor nodes are organized into tree structure. To maintain this structure each node maintains its root node details. The non leaf node performs the aggregation. Some of the aggregation operations could be MIN, MAX, COUNT. Simulators of WSN allows the user to choose the aggregation scheme required[7].

\subsection{Routing Algorithms}

The simulators usually have the stack of algorithms and transmission protocols to choose. It reduces the developers effort in greater extent. Some of the simulators allow the researcher to plug in the algorithm module. It is also of great help. There are simulators like AlgoSIM for evaluating only the algorithms of WSN[8].

\subsection{Layer Modeling}

The simulator must be capable enough to help the developers to visualize all the layers of WSN communication the physical layer, data link layer and the network layer details[9].

\subsection{Transmission Control}

Transmission involves packet formation, address resolution, key generation and localization, parity check to verify the data reception. One example scenario could be the investigator wants to see the content of every packet of the frame in the transmission bit by bit along the frequency monitoring[10]. 


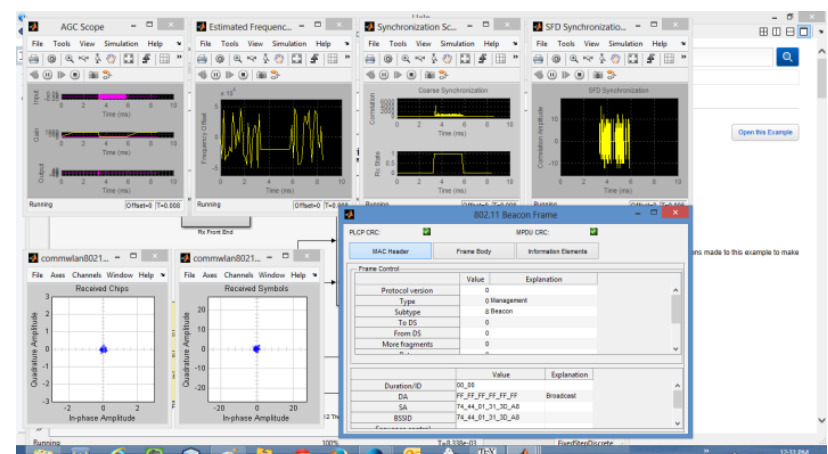

Figure 7: WSN Data Transmission Tracking at the MAC layer in MATLAB

\subsection{Topology Design}

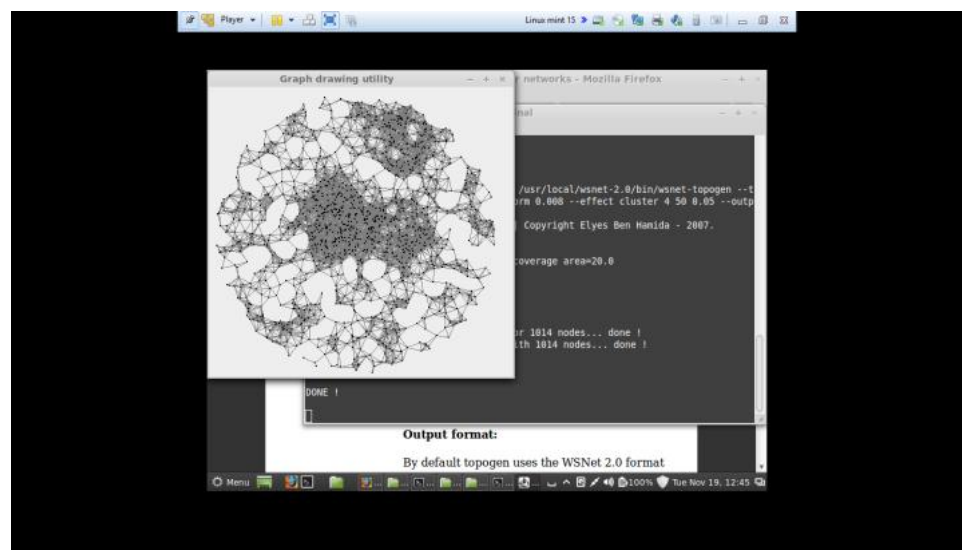

Figure 8: Circular Topology of WSN by WSNET Simulator

In any WSN simulator the researcher must be able to select the topology of the sensors as the data observations vary for different topology[5].

\section{Energy Investigation}

Initial period of Wireless Sensor Network evolved with the goal of better throughput, minimal error packets, minimal retransmission, nearest routing etc. But, later everyone realized that the sensors are of limited power supply and randomly remotely deployed. So, the power supply to the sensors frequently is difficult. There are world wide initiatives in the last decade on "Energy Engineering" discipline[11]. The entire effort can be summarized as follows

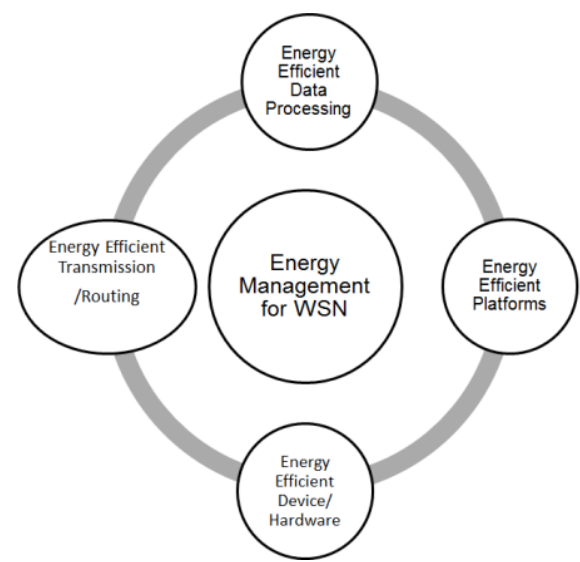

Figure 9: Energy Management in WSN

1. Monitor and control the energy usage in WSN. Eg. The Nodes can be switched to sleep mode when the node is of less use.

2. Address the energy leakages by the energy efficient schemes. Eg. By introducing the energy efficiency correction scheme to the routing algorithm or protocols

3. Energy Harvesting. Eg. Generating energy from the alternate energy sources like solar, wind, vibrant, polar energies. 


\section{Energy Model}

The simulators are also extended to support the investigations of energy model and the energy model relevant developments. Battery model, power spectrum are getting introduced in the simulators. Some of the simulators provide the battery model as a simple framework with the infinite energy concept, it is the programmer responsibility to extend the class and override the methods to decrease the energy per computation or per transmission[12]. Whereas some of the simulators go to the extend of choosing the hardware node type, channel type and gateways. Depending on the selection of WSN profile, the power has been automatically maintained by the application logic.

\section{Challenges}

There are micro details like sensor warm-up, scaling the sensor data, stabilizing the sensors, switching between the states which all involve energy. Even the active state of the node involves the computation activity which is not considered in detail in most of the energy models developed, but only the transmit and sensing energy details are considered in most energy models.

The batteries are of the following types

(i) Analytical models

(ii) Electrical circuit based models

(iii) Stochastic models

(iv) Electrochemical model

The theoretical capacity which is based on the amount of energy stored in the battery, and that is the maximum energy that can be consumed in practice. The standard capacity which is the energy that can be obtained under some conditions specified by the battery manufacturer. The actual capacity which is the amount of energy that the battery delivers under a given load, and is usually used as a metric to judge the battery efficiency. Most of the simulators show the health status means the current power level of the sensor battery.

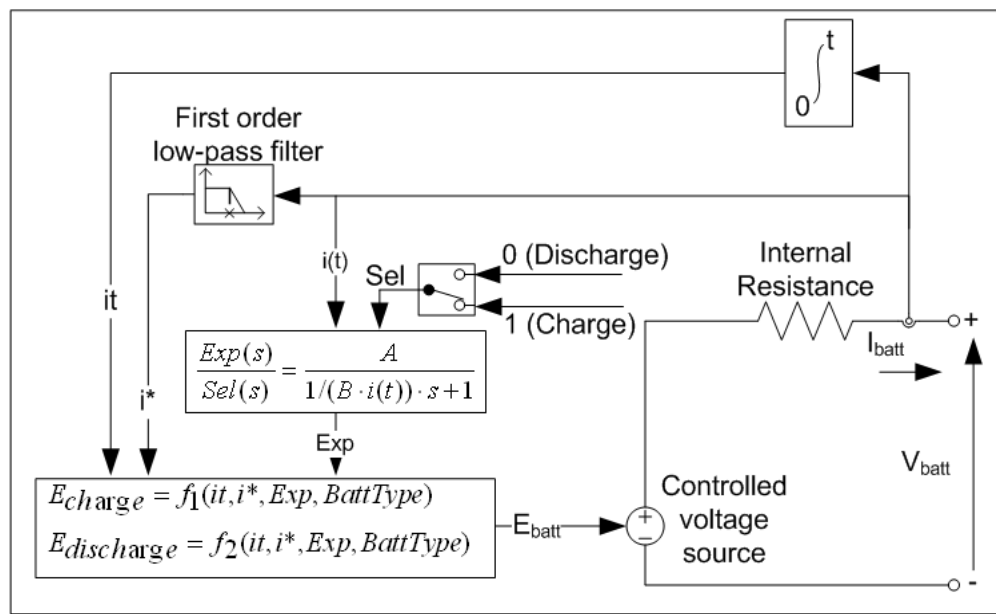

Figure 10: Battery Model in MATLAB

\section{Energy Model Support In Simulators}

Energy can be modeled in WSN with the major software modules: Energy Sources, Energy Stores and Energy Consumers. Each ones behavior is expressed as the methods of the classes[6].

\subsection{Battery Model}

A typical battery model implementation in MAT LAB is discussed here. Most popular rechargeable battery model is considered for the discussion. The detailed chart of information on this battery model is available in the MAT LAB web site and the MAT LAB help manual. Here it is highlighted as a lead[13].

EBatt=Nonlinearvoltage $(\mathrm{V})$

$\mathrm{E} 0=$ Constantvoltage $(\mathrm{V})$

$\operatorname{Exp}(s)=$ Exponential zonedynamics

$\mathrm{Sel}(\mathrm{s})=$ Represents thebatterymode

Sel(s)=0duringbatterydischarge

Sel(s)=1duringbatterycharging.

$i^{*}=$ Lowfrequencycurrentdynamics(A)

$\mathrm{i}=$ Battery current $(\mathrm{A})$

it=Extractedcapacity (Ah) 
$\mathrm{Q}=$ Maximumbatterycapacity $(\mathrm{Ah})$

$\mathrm{A}=$ Exponential voltage $(\mathrm{V})$

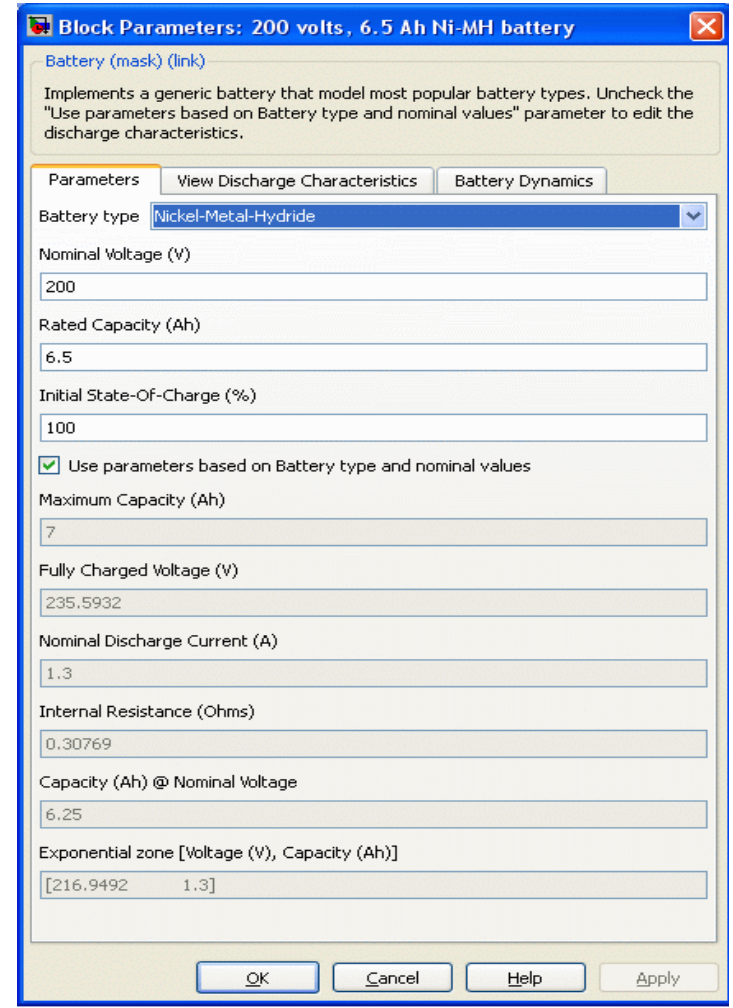

Figure 11: Battery Configuration panel in MAT LAB

\subsection{Energy Frame work in OMNET++}

Herewith the battery class introduced in OMNET++ with the sleep, receive, transmit modes[14].

i. Provision for host failure due to battery drying out

ii. Provision for various energy consumption calculations (CPU, Sensor, Radio etc.)

iii. Assistance for modeling abstract energy operations

iv. Test environment for validation

v. Simple Battery, a simple linear battery model that provides a common interface to all battery using devices.

vi. Battery Stats to collect and format the statistics

vii. Nic80211Battery which extends the mobility-fw Nic80211 to draw power from the battery and goes to shut down mode on host failure.

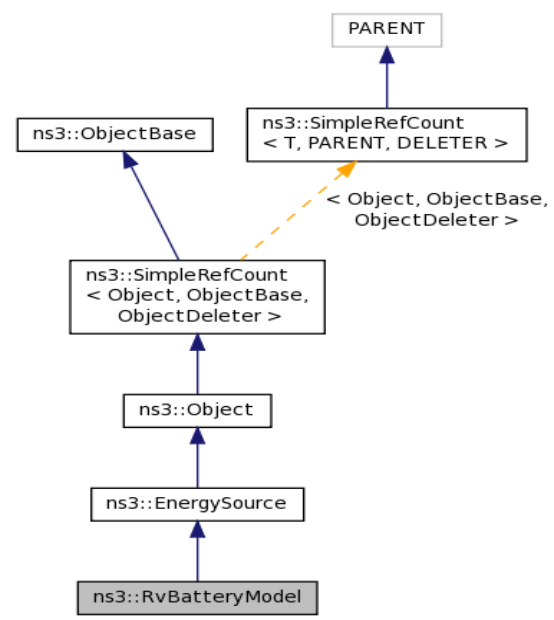

Figure 12:Energy ClassinNS3 


\subsection{Energy Model Reference in NS3}

Most of the details shared here are from the concerned simulators authentic web site for the cross reference. Here the entire energy model with the class hierarchy in NS3simulator has been explained[15].

National Security Lab (NSL) worked on the Energy Model for NS3. The energy model implementation has been developed with the goal to keep track of the energy consumption at every node. It came out with the classes energy source model and device energy consumption model.

Here the energy source class hierarchy has been explained in detail.

\section{Conclusion And Future Direction}

Almost all the simulators discussed has its own energy battery model which collaborates with the other modules on the WSN communications. The battery modules are to be fine tuned for a particular computation energy or state changes which are ignored in some cases[11].

The energy measure trace is not organized in some of the simulators. Except in some of the advanced simulators like MAT LAB, the energy measure improvement is not made sync with the performance[13]. Layer wise, process wise, task wise recording of energy is not clearly detailed out in the Energy data sheet outputs. The energy measures are not detailed with the economics involved with the energy. The better energy portfolios with energy economic measures for different WSN profiles are yet to be developed.

\section{List of Figures}

1 Classification of Simulators 2

2 Factors considered 3

3 Simulator Architecture 3

4 Power Spectrum Graph Generated in MAT LAB $\begin{array}{ll}\text { with Communication Simulation } & 6\end{array}$

5 Designing the Process Flow in MAT LAB 6

6 Signal Propagation Display in MAT LAB simulator 6

7 WSN Data Transmission Tracking at the MAC layer in MAT LAB 7

8 Circular Topology of WSN by WSNET Simulator 8

9 Energy Management in WSN 8

10 Battery Model in MAT LAB 9

11 Battery Configuration panel in MAT LAB 10

12 Energy Class in NS3 11

\section{List of Tables}

$1 \quad$ Features and Architecture

\section{References}

[1] P. S. Rakesh Sharma, S.Kaushik, V. A. Athavale, "simulators for wireless sensor networks: Are view", International Journal of Computer Applications 75(6) (Aug 2013)1-30.doi:0975 8887.

[2] R. Huber, P.Sommer, R. Wattenhofer, "demo abstract: Debugging wireless sensor network simulations with yeti and cooja", ISPN- ACM doi:978-1-4503-0512-9.

[3] H.Sundani, H.Li, V. Devabhaktuni, M.Alam, P.Bhattacharya, Wireless sensor network simulators a survey and comparisons, International Journal of Computer Networks 2(5) (2010)249-265. doi:10.1021/ic9907718.

[4] B. Musznicki, P. Zwierzykowski, Survey of simulators for wireless sensor networks, International Journal of Grid and Distributed Computing 5(3) (September, 2012) 23-50. doi:10.1021/ja0276321.

[5] C. Ptolemaeus, Simulation Design and Modeling, 1stEdition, Creative Commons, 444 Castro Street, Suite 900, Mountain View, California,94041,USA,2014.

[6] Dianemu a java based generic simulation environment for distributed protocols1, Tech.rep., D-76128Karlsruhe, Germany (July, 2003).

[7] L. Girod, M. Csail, N. Ramanathan, Jeremy Elson, T. Stathopoulos, "emstar: A software environment for developing and deploying heterogeneous sensor actuator networks", ACM Transactions on Sensor Networks 3(13) (Aug2007) 1-30. doi:10.1145/1267060.1267061

[8] P.Y. Wang, C.L.Chou, C.C. Lin, The GUI User Manual for the NCTUns 6.0 Network Simulator and Emulator, Network and System Laboratory, Department of Computer Science, National Chiao Tung University, Taiwan (January, 2010).

[9] S.Y. Wang, N. C. T. U. C.-L. Chou, E. T. Chun-Ming Yang, Estinet open flow network simulator and emulator, IEEE Communications Magazine 51 (9)(2013)110-117. doi:0163-6804.

[10] A. Sobeih, W.P.Chen, J.C.Hou, L.C.Kung, N.Li, H.Lim, H.Y.Tyan, H.Zhang, Jsim: A simulation and emulation environment for wireless sensor networks, IEEE Wireless Communicationss13(2005)2006.

[11] J. Eriksson, F. Sterlind, N.Finne, N.Tsiftes, A. Dunkels, T.Voigt, "cooja/mspsim: Interoperability testing for wireless sensor networks", ICST doi:978-963-9799-45-5.

[12] A. D. Fredrik Osterlind, Joakim Eriksson, "demo abstract: Cooja timeline: Apower visualizer for sensor network simulation", ACM Sensysdoi:978-1-4503-0344-6.

[13] [link]. URLhttp://www.mathworks.com

[14] [link]. URLhttp://www.omnetpp.org

[15] [link]. URLhttp://www.nsnam.org 


\section{AUTHORS}

\section{Thangaraj Muthuraman}

$\mathrm{He}$ is an Associate Professor at the Department of Computer Science, Madurai Kamaraj University, Madurai. His research interests are in the area of Data Semantics, Networks and Wireless Sensor Networks. At MKU additionally, he is also a Resource Executive of many academic councils. He has worked on much Government sponsored UGC projects that involve innovation and Domain specific benefits. He has over two decades of experience in the field of Information Technology. He specializes in setting up Global Research and Development and innovation. He is an expert in the area of developing strategies for innovation.

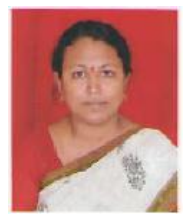

\section{Anuradha Subramanian}

She has Around 12years of software industry experience in Microsoft. Net, J2EE Technologies that involve in analysis, development and integration of Web and Windows based applications, enterprise business servers. Very strong in business process modeling and design. She has work experience in modeling tools, quality processes, Research and Development initiatives Scrum based Agile process methodology. As a Project Manager, manages high powered, fast moving, multi disciplinary development teams. Her research interests are Wireless Sensor Net works and energy harvesting in WSN. She is a research scholar in Madurai Kamaraj University and currently doing her research in the SMART WSN. 